\title{
LA INMUTABILIDAD DE LA GEOPOLÍTICA CLÁSICA. UNA APROXIMACIÓN A LAS RELACIONES ENTRE RUSIA $Y$ LOS ESTADOS UNIDOS*
}

\section{THE IMMUTABILITY OF THE CLASSICAL GEOPOLITICS. AN APPROACH TO THE RELATIONS BETWEEN RUSSIA AND THE UNITED STATES OF AMERICA}

\section{Sebastián Antonino Cutrona*}

\begin{abstract}
RESUMEN
En el marco del Postmodernismo, las bases teóricas fundamentales de la Geopolítica tradicional comenzaron a ser fuertemente cuestionadas por parte de una nueva corriente de investigadores identificados con la Escuela Crítica de esta disciplina. Sin embargo, los diferentes matices en las relaciones entre Rusia y Estados Unidos parecerían haber revalidado las premisas básicas de la Geopolítica tradicional: el Heartland de Halford Mackinder, la Contención de George Kennan y las Esferas de Influencia.

PALABRAS CLAVES: RUSIA * ESTADOS UNIDOS * GEOPOLÍTICA * POLÍTICA EXTERIOR * CONTROL INTERNACIONAL * PETRÓLEO * POLÍTICA DE DEFENSA

\section{ABSTRACT}

Within the frame of postmodernism, the main theoretical bases of the traditional Geopolitics started to be strongly questioned by a new current group of researchers identified with the Critical School of this discipline. However, the different shades in the relations between Russia and the United States of America seem to revalidate the basic premises of the traditional Geopolitics: Halford Mackinder's Heartland, George Kennan's Contention and The Spheres of Influence.
\end{abstract}

KEY WORDS: RUSSIA * UNITED STATES * GEOPOLITICS * FOREIGN POLICY * INTERNATIONAL CONTROL $*$ PETROLEUM $*$ DEFENCE POLICY

Este artículo fue presentado en la cátedra de Geopolítica del Siglo XX de la Maestría Académica en Geografía de la UCR en febrero del 2009.
Profesor regular asistente de la cátedra de Política Internacional, Carrera de Ciencias Políticas, Universidad Nacional de La Rioja (UNLaR). sebastiancutrona@hotmail.com 


\section{INTRODUCCIÓN}

La década de los noventa marcó una bisagra en la configuración del escenario internacional. El derrumbe de la Unión de Repúblicas Socialistas Soviéticas (URSS) y la desaparición de los socialismos reales, provocó la desarticulación del sistema bipolar y el viejo orden internacional confeccionado posteriormente a la Segunda Guerra Mundial (II GM). Estos acontecimientos no tardaron en repercutir fuertemente en la comunidad académica; disciplinas como las Relaciones Internacionales, la Ciencia Política, la Geografía Política, entre otras, trataron rápidamente de brindar explicaciones frente a la nueva arquitectura internacional; aunque en esta ocasión, quizá sin la rigurosidad teórica característica de otros tiempos. Autores como Francis Fukuyama (1992), plantearon que la desaparición del coloso socialista había terminado por imponer definitivamente la democracia occidental, $y$ con ella el capitalismo en lo que él denominó "El fin de la Historia y el último hombre"; Samuel Huntington (2007), fue un poco más allá al proyectar un nuevo orden internacional, donde los principales conflictos no serían protagonizados por unidades estatales, sino por "El choque de civilizaciones". Si bien estos autores no agotan, ni mucho menos, la nueva oferta intelectual a la que hacemos referencia, decidimos exponerlos como representantes, debido al grado de popularidad y trascendencia con la que han contado.

Dentro de este marco y adentrándonos más en la temática que nos compete, también muchos de los supuestos de la Geopolítica clásica, como era de esperar, sufrieron un fuerte revés posteriormente a la finalización de la Guerra Fría. El nuevo statu-quo le arrebató a esta disciplina el sustento práctico del que había gozado durante mucho tiempo. El nuevo escenario internacional "Post Guerra Fría", llevó a muchos analistas a plantear la necesidad de promover un cambio paradigmático en la Geopolítica. En esta dirección, una nueva corriente de investigadores buscaron $-y$ continúan haciéndolo- deconstruir esta disciplina, promoviendo un inédito re-pensar en esta materia. Estamos hablando, fundamentalmente, de los partidarios de la Geopolítica Crítica, la cual "forma parte del giro postestructural que se ha producido en la geografía humana" (Agnew, Flint, 2002: 111). John Agnew, Gearóid Ó Tuathail, Simon Dalby, entre otros, constituyen actualmente los principales representantes de esta tendencia postmodernista.

Sin embargo, el devenir de diferentes acontecimientos internacionales en la actualidad, parecen haberle quitado cierto vigor a estas iniciativas. Entendiendo que la teoría adquiere mayor rigurosidad cuando la realidad se manifiesta concomitantemente a los supuestos que esta establece, se podría argumentar que la Escuela Crítica habría fallado al menos en un aspecto: no se habría producido, por lo menos hasta ahora, una modificación en la forma de concebir la geografía; es decir, los patrones de pensamiento continuarían siendo los mismos.

Es que lejos de haber sido completamente sepultados, muchos de los supuestos de la Geopolítica clásica parecen ser objeto de una nueva revalidación empírica. En esta dirección, el objetivo de este trabajo será contrastar analíticamente las premisas básicas de la Geopolítica tradicional a la luz del, a priori, nuevo orden internacional en configuración. Con base en este cometido, y sin la ambición de alcanzar explicaciones absolutas y representativas de todo el sistema, ni mucho menos extrapolables, utilizaremos los diferentes matices en la evolución de las relaciones ruso-estadounidenses al finalizar esta década como unidad de análisis básica para revalidar, o no, la aplicabilidad de los conceptos elaborados por los denominados "clásicos de la Geopolítica". Verbigracia, la Teoría del Corazón Continental (Heartland) de Sir Halford Mackinder, la Teoría de la Contención de George Kennan y el paradigma de las Esferas de Influencia, constituirán la base teórica medular para analizar la supuesta reconfiguración del escenario internacional y la viabilidad práctica de los postulados tradicionales.

¿Continúan las grandes potencias obrando internacionalmente de acuerdo a los postulados de la Geopolítica clásica? ¿La Geopolítica clásica es la única herramienta válida para entender la realidad? ¿Se está produciendo un 
retorno a los clásicos o es que nunca se fueron? ¿Es imposible pensar en la emancipación de los conceptos clásicos de esta disciplina? ¿La Geopolítica clásica seguirá presente en la medida en que sus postulados sean funcionales a los intereses de las potencias? ¿Estas premisas fundamentan una nueva edición de la Guerra Fría? ¿Son la clara manifestación de las ambiciones imperialistas de Rusia y Estados Unidos? Estas preguntas, $y$ muchas otras más, nos servirán como guía y estrella polar en el desarrollo de este trabajo.

\section{CAMBIO DE ORDEN INTERNACIONAL}

Si adoptamos la clásica categorización desarrollada por Robert $\mathrm{Cox}^{1}$, la última década del siglo xx fue testigo de un cambio de orden internacional. Sin lugar a dudas, la caída del muro de Berlín (1989), la disolución de la Unión Soviética (1991) y la progresiva emancipación de los Estados "satélites", fueron las insignias principales del tránsito hacia una nueva era. El sistema de jerarquías estatales, las estrategias, los procedimientos y las instituciones internacionales, sufrieron fuertes alteraciones durante el transcurso de estos años. Sin embargo, el nuevo escenario internacional no exhibía para ese entonces, la estabilidad necesaria para estructurar una nueva clasificación axiomática del sistema; solo un par de asuntos resultaron prácticamente incuestionables: Estados Unidos había conseguido imponerse formalmente - aunque tácitamente este hecho se había consumado varios años atrás - a la Unión Soviética, y de esta manera, el modelo económico capitalista sobre el arquetipo de planificación centralizada. Grosso modo, la nueva ingeniería internacional se caracterizó por la primacía de los Estados Unidos, la constitución de diferentes bloques económicos y

1 R. Cox plantea que los órdenes mundiales tienen una estrecha relación con la hegemonía de un Estado particular. En este sentido, el hegemón establece un orden mundial y luego lo protege mediante la creación de diferentes instituciones internacionales de diverso carácter: económicas, políticas, sociales, etc. políticos dentro del marco del regionalismo abierto, el afianzamiento progresivo del capitalismo, el crecimiento económico explosivo de naciones antaño subdesarrolladas, la expansión del comercio internacional, la acentuación de la polarización económica mundial, entre otros.

Sin embargo, los atentados terroristas del 11 de septiembre del 2001 (11-S), modificaron rápidamente esta tendencia. Un grupo de extremistas, armados con navajas y utilizando el propio sistema de transporte norteamericano, alcanzaron exitosamente lo que muchos otros no pudieron lograr durante siglos: poner en jaque la seguridad de la potencia militar más grande del planeta. Es evidente que la génesis de esta nueva gama de actores internacionales, dotaría al sistema de características difícilmente previstas con anterioridad. El clásico esquema interestatal de origen westfaliano, ya no sería adecuado para explicar la nueva lógica de las "relaciones internacionales". Estas tendencias parecían indicar que la nueva ingeniería internacional había evolucionado en una dirección completamente novedosa; donde los conflictos simétricos, las alianzas militares interestatales, el ejercicio del poder disuasivo, entre otros, ya no serían más representativos de esta era. Frente a este nuevo panorama, la estrategia norteamericana se inclinó enérgicamente a la "cruzada contra el terrorismo"; una tarea que llevaría al país anglosajón a adoptar una política exterior de carácter eminentemente hostil. Numerosas invasiones e intervenciones internacionales fueron justificadas con esta finalidad.

Probablemente, nadie hubiese podido imaginar un escenario radicalmente distinto al descrito ut supra. Es decir, el terrorismo había pasado a ocupar el nuevo rol de enemigo internacional y ningún otro organismo se perfilaba claramente como una seria amenaza para el equilibrio del sistema patrocinado por los Estados Unidos. Es que más allá de las tímidas $y$ ambiguas manifestaciones contra-hegemónicas de China, Cuba, Irán, Irak y Corea del Norte, ningún Estado había logrado desafiar la supremacía norteamericana hasta el punto de poner en peligro la estabilidad del sistema. En contra de casi todos los pronósticos, no 
sería el terrorismo esta vez quien inquietara a los agentes de seguridad del gobierno de los Estados Unidos, sino su histórico y tradicional contendiente: Rusia.
En la tabla siguiente podemos observar sintéticamente el proceso evolutivo de los diferentes órdenes internacionales posteriormente a la II GM de acuerdo con los principales actores del sistema.

TABLA

ÓRDENES INTERNACIONALES

\begin{tabular}{lllll}
\hline PERÍODO & 1947-1991 & 1991-2001 & $2001-2008$ & $2008-?$ \\
\hline $\begin{array}{l}\text { Tipo de orden } \\
\text { Internacional }\end{array}$ & $\begin{array}{l}\text { Guerra Fría } \\
\text { (Bipolar) }\end{array}$ & $\begin{array}{l}\text { Trasnacionalismo } \\
\text { liberal (Unipolar) }\end{array}$ & $\begin{array}{l}\text { Guerra asimétrica } \\
\text { (Unipolar) }\end{array}$ & $\begin{array}{l}\text { II Guerra Fría? } \\
\text { (Bipolar?) }\end{array}$ \\
\hline Actores principales & Estados Unidos-URSS & Estados Unidos & $\begin{array}{l}\text { Estados Unidos-enemigos } \\
\text { de tercera generación }\end{array}$ & $\begin{array}{l}\text { Estados } \\
\text { Unidos-Rusia? }\end{array}$ \\
\hline
\end{tabular}

Fuente: autor.

\section{LA RESURRECCIÓN RUSA}

Todo parecía indicar que el clásico adversario norteamericano había decidido retirarse definitivamente de la primera plana mundial. Una economía empobrecida, una corrupción galopante, y por sobre todo, un crecimiento militar insostenible - overstretch-, habían terminado de minar la vitalidad de Rusia. Sobre sus ruinas, se erigió la Comunidad de Estados Independientes (CEI), una nueva configuración institucional que concentró a gran parte de las ex Repúblicas Socialistas Soviéticas ${ }^{2}$, aunque en este caso, ya no bajo el clásico y férreo control moscovita.

Los problemas internos que debía afrontar el Kremlin, eran suficientes como para pensar en un resurgimiento militar e ideológico tan fuerte como el que había tenido durante la Guerra Fría. Sin embargo, una serie de maniobras llevadas a cabo por el gobierno de los Estados Unidos, sobrepasaron una línea que Rusia, esta vez, no estaba dispuesta a tolerar: (I) la instalación de un escudo antimisiles en Polonia, (II) el establecimiento de un complejo sistema de radares en la República Checa (III) $y$ el posible ingreso - patrocinado por Estados

Diez de las antiguas quince Repúblicas forman parte de la CEI. Solo los tres países Bálticos (Estonia, Letonia y Lituania) no adhirieron al tratado, más Turkmenistán y Georgia que se retiraron posteriormente.
Unidos - de Georgia, Ucrania y otras naciones integrantes de la ex URSS, a la Organización del Tratado Atlántico Norte (OTAN). Si bien estos acontecimientos desencadenaron directamente el abandono del letargo ruso, otra serie de factores favorecieron esta tendencia: (IV) el brusco crecimiento de los precios internacionales de los bienes primarios; principalmente el petróleo ${ }^{3}$, lo cual favoreció rápidamente la reactivación de su economía; y (v) la llegada al poder de una renovada elite política dispuesta a restaurar la clásica política imperialista rusa. Posiblemente, la ausencia de uno de estos elementos hubiese modificado la orientación de la política exterior moscovita en la actualidad, hasta el punto de que hoy no estaríamos en condiciones de hablar del acelerado retorno del país a la arena internacional.

Detrás de cada actitud de reivindicación y/o censura entre ambas potencias, subyace una visión estratégica $y$ una forma en que cada una de ellas concibe el espacio y la geografía; constituyéndose, al fin y el cabo, en las bases de los respectivos códigos geopolíticos de cada nación. Hacia ellos dirigiremos nuestra mirada, porque entendemos que más allá de toda retórica, los códigos geopolíticos constituyen los ejes fundamentales sobre los cuales se diagrama la política exterior de todos los Estados.

3 Alcanzando un máximo histórico de 143, 57 dólares el barril de crudo, precio que fue rebasado en las siguientes transacciones electrónicas. 


\section{CÓDIGOS GEOPOLÍTICOS}

Siguiendo a J. Gaddis (1982), los códigos geopolíticos son supuestos geográfico-políticos sobre los cuales se basa la política exterior de un país. Deben definir los intereses del Estado, identificar las amenazas y planificar la respuesta ante cada una de ellas. Si bien existen tantos códigos como países, la trascendencia es considerablemente mayor cuando estos pertenecen a las grandes potencias, en la medida que tienen la capacidad de influenciar los respectivos códigos geopolíticos del resto de las naciones del planeta. Pero es verdad que no todos los Estados han tenido la misma capacidad para llevar a cabo sus visiones geopolíticas. Solo las Grandes Potencias de cada era han logrado inscribir sus particulares imaginaciones geopolíticas en el conjunto del mundo (Agnew, 2005a).

La renovada rivalidad ruso-estadounidense se encontraría cimentada en la usanza de una serie de códigos geopolíticos específicos, los cuales se han visto reiteradamente influenciados por las teorías geopolíticas clásicas. En esta exposición, solamente nos limitaremos a estudiar los autores pertenecientes a la denominada "Geopolítica tradicional", debido a que entendemos que la política exterior de las grandes potencias se seguiría guiando - o utilizando según el pensamiento de John Agnew- por sus postulados claves. Se expondrán separadamente a fines puramente analíticos, puesto que en la realidad sería imposible realizar una abstracción individual de alguno de ellos; es decir, las teorías y las doctrinas geopolíticas suelen entremezclar su influencia en la conformación de las políticas exteriores de un país en un período de tiempo determinado.

\section{HEARTLAND}

Publicada en 1904, la clásica tesis del "Pívot Geográfico de la Historia" de Halford Mackinder, constituyó una de las principales influencias teóricas en la política exterior de las grandes potencias durante el transcurso de la Guerra Fría. Sintéticamente, en esta obra, Mackinder diseña una rígida jerarquización del espacio geográfico mundial; verbi- gracia, identifica a la región asiática central como la "región pivote" del mundo, inaccesible para cualquier potencia marítima de la época, la cual se encuentra rodeada por un "cinturón interior" (inner crescent) en Asia y Europa continental, y por un "cinturón exterior" (outer crescent) conformado por el resto del territorio mundial. Claramente, en estos escritos subyace el miedo ante una posible alianza entre Rusia y Alemania, lógicamente, en detrimento de Gran Bretaña. Sin embargo, en una revisión posterior (1919), el geógrafo inglés introduce una serie de modificaciones a su modelo original. En la nueva versión, Asia Central se constituye como el Corazón Continental (Heartland), el cual adquiere una extensión sensiblemente mayor. Sin embargo, se mantienen la misma estructura y el temor a que Alemania domine el Corazón Continental (Taylor, 2002). La lógica de esta clasificación y la jerarquía de cada una de estas regiones, queda visiblemente plasmada en una de sus premisas más trascendentales:

Quien gobierne la Europa Oriental dominará el corazón continental; quien gobierne el corazón continental dominará la isla mundial; quien gobierne la isla mundial dominará el mundo. (Mackinder en Taylor, 2002: 58).

Si bien, como mencionamos anteriormente, esta tesis tuvo un fuerte impacto en la orientación de la política exterior de las potencias durante la Guerra Fría, ciertos indicios parecen indicar que en la actualidad la situación no sería radicalmente distinta. Para poder comprobar, o no, esta hipótesis, debemos prestar detenidamente atención a ciertos sucesos internacionales que han tenido lugar durante los últimos tiempos. Curiosamente, las maniobras militares $y$ diplomáticas norteamericanas más trascendentales para el sistema mundial, posteriormente al 11-S, coinciden geográficamente con la región del "cinturón interior" de Mackinder. Análogamente, ha sido el enclave donde Rusia ha manifestado mayor resistencia e intransigencia ante los continuos avances de los Estados Unidos. Veamos esto más detalladamente: 
a. Una de las consecuencias militares del 11-S fue la invasión de Afganistán. Supuestamente con el objetivo de atrapar al principal actor involucrado en los ataques -Osama bin Laden-, el 7 de octubre del 2001 el ejército de los Estados Unidos activa la campaña "Operación Libertad Duradera", luego de que el Gobierno acusara al régimen Talibán Afgano de ocultar al principal sospechoso de los atentados. Si consultamos cualquier mapa político, podremos observar que Afganistán se encuentra justamente ubicado en la frontera sur de la región pivote, con una gran parte del territorio emplazado dentro del "cinturón interior".

b. El 19 de marzo del 2003, un conjunto de fuerzas militares lideradas por el gobierno de los Estados Unidos, invade Irak. Aparentemente con el objetivo de "desarmar a Irak de armas de destrucción masiva (ADM), poner fin al apoyo brindado por Saddam Hussein al terrorismo, y lograr la libertad al pueblo iraquí" (Bush, The White House, march, 2003), las tropas bombardearon las principales ciudades del país. Tal cual el ejemplo anterior, Irak también se encuentra ubicado dentro del "cinturón interior" y es la continuación del mismo, hacia su extremo izquierdo.

c. Un poco más adelante en el tiempo, el 7 de julio del 2008, el gobierno de la República Checa y los Estados Unidos, por medio de Condoleezza Rice y su homólogo, Karel Schwarzenberg, firman un acuerdo básico para la instalación de un complejo sistema de radares norteamericanos en las montañas de Brdy. Curiosamente, la República Checa también se encuentra ubicada dentro del "cinturón interior".

d. Como parte de la misma estrategia, el 20 de agosto del 2008, Estados Unidos y Polonia, a través de Condoleezza Rice y el Ministro Polaco de Asuntos Exteriores, Radoslaw Sikorski, suscriben un acuerdo para la instalación de un escudo antimisiles en Polonia ${ }^{4}$. Se estima que la lanzadera de misiles de

$4 \quad$ Al igual que en la instalación de los radares en la República Checa, el poder legislativo de Polonia debe aprobar el acuerdo para que la medida se haga completamente efectiva. alcance medio tipo "Patriot", estará completamente finalizada para el 2012 y estará ubicada en el norte del país. Tal cual los tres primeros ejemplos, Polonia se sitúa claramente en el margen occidental del "cinturón interior".

e. Quizá como una consecuencia lógica de las incursiones norteamericanas a pocos kilómetros del territorio ruso, el Kremlin amenazó recientemente con instalar una base misilística en Kalingrado, un enclave ruso limítrofe con Polonia. Este complejo militar permitiría bloquear electrónicamente el sistema estadounidense, que se desplegará en Polonia y la República Checa.

f. En el nivel netamente diplomático, el gobierno de los Estados Unidos ha bregado constantemente por el ingreso de Ucrania y Georgia, ex repúblicas socialistas soviéticas, a la OTAN. Ambos Estados se encuentran ubicados dentro del "cinturón interior" y han sido tradicionalmente considerados por Rusia, como aliados claves bajo su esfera de influencia directa.

g. El conflicto desatado en la República de Georgia más recientemente, coincide directamente con esta tendencia. La declaración de independencia de Osetia del Sur y Abjasia en agosto del 2008, provocó la intervención directa de Rusia en la contienda. Paralelamente, el gobierno de los Estados Unidos, por medio de la OTAN, intercedió en numerosas ocasiones con el objetivo de contrarrestar la influencia del Kremlin en la región.

h. Por último, a comienzos del 2009 el conflicto ruso-ucraniano por el transporte de gas moscovita por el territorio ucraniano amenazó con exponer a Europa a las crudas condiciones del invierno. Luego de fuertes acusaciones cruzadas entre ambos gobiernos, Rusia $y$ Ucrania debieron aceptar la intervención de la Unión Europea para frenar la escalada del conflicto. El acontecimiento tuvo su punto más álgido cuando Moscú decidió cortar por completo el suministro de gas con destino a Europa, tras acusar a Kiev de robar y desviar el recurso. Situación que motivó a interceder al vértice europeo para evitar que la disputa impacte más fuerte en Europa.

Muy probablemente estemos omitiendo inconscientemente algún acontecimiento 
en esta lista, sin embargo, la magnitud de los sucesos enumerados ut supra, no pueden pasar fácilmente inadvertidos. En todas estas ocasiones, Estados Unidos y Rusia se han enfrentado en forma indirecta, con el objeto de garantizar un dominio relativo sobre la región. Resulta evidente, que esta zona - "cinturón interior"ha sido históricamente clave para las potencias mundiales, $y$ en este caso, los hechos han confirmado esta premisa. Más allá de la riqueza en recursos naturales ${ }^{5}$, esta zona del planeta parece tener un sentido simbólico especial para ambos Estados, que dista mucho de sus características meramente geográficas. A veces, "la importancia del lugar no se deriva de ninguna localización especial ni de su riqueza en recursos, sino que es una construcción social histórica" (Cairo en Agnew, 2005a: XIV). Naturalmente, el "cinturón interior" ha adquirido una preponderancia significativa, debido a que la titularidad de la "región pivot" parece estar fuera de toda discusión por el momento. Por el lado de Rusia, el "cinturón interior" comienza paralelamente con la culminación de su propio territorio; sin embargo, el Kremlin también ha considerado a algunas de las naciones allí ubicadas, como una extensión continental natural de su espacio soberano. Por el otro lado, Estados Unidos ha buscado legendariamente, por vías diplomáticas y militares, limitar y sujetar la influencia moscovita en su propio hinterland (Fig. 1). Esta maniobra estadounidense en la

\section{FIGURA 1}

\section{INTERVENCIONES NORTEAMERICANAS EN EL "CINTURÓN INTERIOR" POSTERIORMENTE AL 11-S}

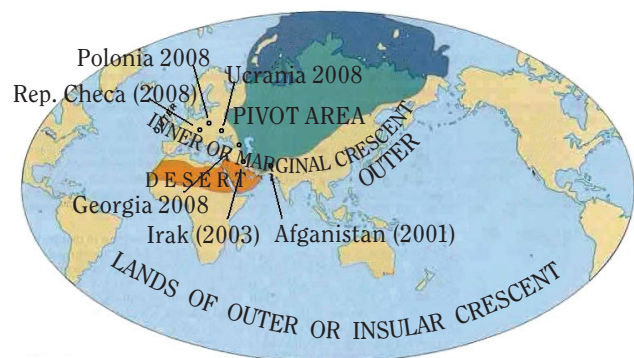

Fuente: Autor. región, como veremos en el acápite siguiente, ha formado parte de la denominada Teoría de la Contención.

\section{LA CONTENCIÓN}

La brusca expansión de la URSS durante $y$ posteriormente a la II GM, provocaron una profunda alarma en el gobierno de los Estados Unidos. La desaparición de la amenaza nazi y los "países del eje" ya no justificaban la alianza soviético-americana confeccionada durante la guerra. Paralelamente, Gran Bretaña comenzaba paulatinamente a manifestar los últimos signos vitales de un imperio en vías de extinción. En su lugar, Estados Unidos asomaba como el artífice principal de la gran victoria. Con una economía en pleno apogeo, la nación menos perjudicada por los estragos de la guerra, se perfilaba como el sucesor natural a ocupar el espacio dejado por el imperio británico. Del otro lado de la vereda, el triunfo de la URSs había sido parcialmente opacado por los graves daños sufridos durante el proceso de la guerra. Sin embargo, esto no representaría un obstáculo serio para las nuevas ambiciones del Kremlin.

Rápidamente, las reuniones sucesivas a la finalización de la guerra, manifestaron claramente que la convivencia entre las dos superpotencias no sería nada fácil. Las divergencias eran cada vez más evidentes. Al cabo de un par de años, los principales responsables del triunfo en la II GM, tuvieron que redireccionar rápidamente sus respectivas políticas exteriores en detrimento de su antiguo aliado. Estados Unidos, tuvo que abocarse ágilmente a la tarea de encontrar un método que le permitiera contrarrestar la creciente influencia de la URSS en todo el planeta. Las bases teóricas de esta nueva estrategia, procedieron de los escritos de George Kennan. En el famoso "Telegrama Largo", el diplomático norteamericano en la URSS, elaboró las bases de lo que a la postre sería una de las doctrinas con mayor influencia en la historia de la política exterior estadounidense: "La

5 Rusia, Irak, Kazajstán son grandes productores de petróleo. Sin embargo, se estima que otras

naciones de la región tienen un potencial en hidrocarburos inconmensurable. 
Doctrina de la Contención". Aunque con pequeños altibajos, esta estrategia fue implementada prácticamente durante todo el transcurso de la Guerra Fría.

Ambigua en muchos de sus postulados, la Teoría de la Contención planteaba que debido a las propias características del régimen soviético $^{6}$, no era aconsejable que el gobierno de los Estados Unidos enfrentara directamente a su adversario. Por el contrario, Kennan establecía una serie de medidas fundamentales para garantizar la victoria norteamericana:

... no es posible oponérsele con eficacia con actos esporádicos que representan los caprichos momentáneos de la opinión democrática, sino sólo por medio de políticas inteligentes de largo alcance por parte de los adversarios de Rusia, políticas no menos estables en sus propósitos, $y$ no menos variadas e ingeniosas en su aplicación, que las de la propia Unión Soviética. En estas circunstancias, es evidente que el elemento principal de cualquier política estadounidense hacia la Unión Soviética puede ser una contención a largo plazo, paciente pero firme $y$ vigilante, de las tendencias expansionistas rusas... (Kennan, 1947).

Al igual que gran parte de sus colegas en Estados Unidos, el pensamiento de George Kennan, muy posiblemente se haya organizado sobre las bases de la clasificación geográfica concebida por Halford Mackinder.

Aunque no hay pruebas de que existiera una conexión directa entre Mackinder y Kennan, si es cierto que las ideas de Mackinder, aunque pensadas para un contexto histórico muy diferente, adquirieron

Durante su estadía oficial en la URSS, Kennan aseguraba haber identificado las características fundamentales del comportamiento soviético. Entre ellas, el diplomático estadounidense, resaltaba la flexibilidad, la cautela, la circunspección y el engaño. Según él, estas consideraciones hacían del régimen soviético un adversario mucho más peligroso que el propio Hitler y/o Napoleón. entre algunos intelectuales de la seguridad estadounidense de la posguerra un carácter profético pero científico para naturalizar la contención como política exterior y militar. (Agnew, 2005a: 132).

Pero a diferencia de Mackinder, los escritos de George Kennan carecen de una ejemplificación geográfica exhaustiva. A pesar de ello, el denominado "arquitecto de la Guerra Fría”, estableció ciertas consideraciones que sí tuvieron implicancias claramente territoriales. Kennan argumentaba que los Estados Unidos se había garantizado el control de cuatro de los cinco centros industriales más desarrollados de la época ${ }^{7}$, lo cual era vital para afianzar la supremacía norteamericana sobre la URSS. Pero es aquí justamente donde se produce una ruptura con el pensamiento mackinderiano: Kennan consideraba a Estados Unidos, Japón, Inglaterra, Alemania y la URSS como enclaves geopolíticos vitales, puesto que en sus territorios se ubicaban los polos industriales más importantes del planeta. De todos ellos, Estados Unidos dominaba los cuatro primeros, por lo tanto, la URSS conservaba únicamente uno (el propio). Podemos observar, que mientras Kennan incluía a Estados Unidos, Gran Bretaña y Japón dentro de esta pentarquía geopolítica; en los escritos de Mackinder, por el contrario, estas naciones forman parte del "cinturón exterior".

Aunque revitalizada durante la presidencia republicana de Ronald Reagan, la política de la Contención pareció desvanecerse conjuntamente con la desintegración de la URSS. Sin un enemigo que contener, las bases de esta doctrina resultaban completamente obsoletas. Sin embargo, actualmente una serie de acontecimientos internacionales parecen indicar que el gobierno de los Estados Unidos habría decidido recurrir nuevamente a muchas de sus premisas fundamentales.

a. El posible ingreso de las antiguas repúblicas soviéticas a la OTAN, es la manifestación más

Estados Unidos, Japón, Inglaterra, Alemania y la URSS. Salvo este último, Estados Unidos tenía el control de todos ellos. 
evidente de los Estados Unidos, en su ambición de contrarrestar la recuperación de la influencia rusa en la región. El hipotético ingreso de Ucrania y Georgia a la organización militar, podría significar una extensión de la influencia norteamericana en Eurasia, además de contener el posible avance ruso hacia occidente. Esta medida no solo erigiría una barrera política en el margen oriental de Europa, sino que también limitaría el acceso de Rusia al Mar Negro. Paralelamente, Estados Unidos se garantizaría una excelente posición en la ruta del transporte energético mundial.

b. El emplazamiento del sistema de radares en la República Checa, también constituye un obstáculo militar para las posibles ambiciones de Rusia en la región. Más aún, si consideramos a República Checa como la "frontera natural" entre Europa y el continente asiático, la presencia militar de Estados Unidos puede constituirse como un cerrojo político-militar clave para obstaculizar cualquier maniobra rusa en la región.

c. La instalación de la base misilística norteamericana a escasos kilómetros del mar Báltico, puede ser claramente interpretado como una medida de contención militar. Los silos norteamericanos en Polonia, supuestamente estarían en condiciones de interceptar cualquier ataque proveniente desde Irán. Sin embargo, y más allá de toda retórica, es evidente que el montaje del complejo sistema defensivo a pocos kilómetros del territorio ruso, tiene como objetivo principal disuadir al Kremlin y desequilibrar la balanza de poder a favor de Norteamérica. Además, esta maniobra le otorgaría indirectamente al gobierno estadounidense cierto control sobre el mar Báltico, con todo lo que ello implica.

d. El apoyo norteamericano a Tiflis, la capital georgiana, durante las tentativas separatistas de Abjasia y Osetia del Sur, es una clara exhibición del interés de los Estados Unidos en limitar la influencia rusa en el sector. Lógicamente, las operaciones norteamericanas, en esta ocasión, solo se cristalizaron bajo el paraguas de la OTAN. Una incursión directa en el antiguo territorio soviético y aún considerado como su "patio trasero", hubiese agravado aún más la situación. Sin embargo, a diferencia de los casos anteriores, en esta ocasión, el gobierno norteamericano atravesó una línea que Rusia no estaba dispuesta a tolerar. Esta vez, la injerencia de Estados Unidos en el Mar Negro, considerado como parte del propio hinterland ruso, fue percibido como una "amenaza directa" por parte del Kremlin. Como resultado, la política exterior moscovita, como veremos más adelante, marcó un quiebre desde este momento, emprendiendo una dirección radicalmente distinta.

e. Por expresa recomendación de George W. Bush, el gobierno de los Estados Unidos decidió retirar de la consideración del Congreso, el acuerdo para la cooperación civil nuclear con Rusia; otra maniobra de notoria hostilidad contra el Kremlin. Según analistas del New York Times, esta medida puede costarle a Rusia billones de dólares en ganancias potenciales. Es que más allá de las importantes consecuencias diplomáticas de esta medida,

... the agreement would have cleared the way for extensive commercial nuclear trade, technology transfers and joint nuclear research between the two countries. And it would have allowed Russia to move forward with the lucrative business of importing, storing and possibly reprocessing spent nuclear fuel from United States-supplied reactors around the world. (New York Times, 8 de septiembre del 2002).

f. Aunque cristalizados previamente al actual acercamiento diplomático de Rusia y Latinoamérica, Estados Unidos ha promovido durante los últimos años una serie de acuerdos con países de América Latina y el Caribe: el Plan Colombia, la Iniciativa Mérida, los Tratados de Libre Comercio (TLC), entre otros. Una estrategia que también busca consolidar la influencia norteamericana en el continente, aunque en esta ocasión, por medios institucionales. 
Tal cual pudimos observar, el gobierno de los Estados Unidos ha ido desarrollando progresivamente una serie de medidas militares, económicas y diplomáticas, que en su configuración final, coinciden con las premisas fundamentales de la clásica Teoría de la Contención. Si bien resulta evidente que muchas de las acciones de Washington tienen como objetivo limitar o contener el nuevo avance ruso, esta región también posee innumerables recursos naturales, que seguramente, serán vitales para la economía norteamericana en el futuro.

\section{ESFERAS DE INFLUENCIA}

Una de las particularidades más representativas de la Guerra Fría fue que ninguna de las dos superpotencias llegó a enfrentarse directamente en el campo de batalla. Todos los conflictos armados tuvieron como involucrados directos a terceras naciones en territorios apartados. Sin embargo, el papel de las superpotencias no fue secundario, ni mucho menos, su rol consistió fundamentalmente en movilizar las voluntades de los gobiernos bajo su influencia, suministrar apoyo económico, militar y diplomático ahí donde sus intereses estuvieran en juego. Así fue que el planisferio político comenzó a ser rediseñando de acuerdo con la disposición de cada nación respecto a las dos superpotencias: por un lado, aquellos países que se encontraban bajo la órbita de influencia de los Estados Unidos; por el otro, los Estados subsidiarios del régimen soviético; entre ellos, un grupo de países que decidió no adherir a ninguno de los dos bloques, las denominadas "naciones no alineadas".

En un mundo completamente polarizado, donde la afinidad con uno de los bloques, irremediablemente constituía la razón fundamental para convertirse en el enemigo del otro, la lógica imperante fue la del "juego de suma cero"; es decir, la alianza de una potencia con un nuevo Estado, era percibida como una pérdida para su adversario. Esta obsesión con la ganancia relativa en la competición interestatal es consustancial con la imaginación geopolítica clásica (Agnew, 2005a). Por ello, la estrategia de las dos superpotencias tenía como objetivo ampliar progresivamente su esfera de influencia, limitando de esta manera la de su enemigo. Sin embargo, este procedimiento, en principio, no tiene un sustento teórico tan evidente como en los dos casos anteriores. Más bien, en este caso estaríamos en condiciones de hablar de la propia esencia de la Guerra Fría en sí misma. En un sentido amplio, numerosas teorías y prácticas de política exterior ${ }^{8}$ válidamente reconocidas, han contribuido conjuntamente en la estructuración de este patrón de comportamiento internacional.

Tal cual los primeros tres acápites, intentaremos demostrar que actualmente las políticas exteriores de Estados Unidos y Rusia respectivamente, tendrían nuevamente como una de sus metas principales, consolidar su influencia sobre terceras naciones. Naturalmente, en este caso, a diferencia de lo sucedido durante la Guerra Fría, la influencia de Rusia y Estados Unidos no se encuentra fundamentada en la ideología capitalista o comunista, ya que en la actualidad no existe tal divergencia.

a. La instalación del escudo antimisiles en Polonia, al igual que el emplazamiento del complejo sistema de radares en la República Checa, es una clara manifestación de la ambición norteamericana por extender su

En Estados Unidos, serían plausibles de ser incluidas dentro de este grupo, la "Doctrina Monroe" elaborada por John Quincy Adams y atribuida a James Monroe; el "Corolario Roosevelt" por parte del presidente de los Estados Unidos Theodore Roosevelt; la "Doctrina del Destino Manifiesto"; la "Diplomacia del Dólar" del presidente William Howard Taft; la "Doctrina Truman" del presidente Harry S. Truman; la "Teoría del Efecto Dominó" de Dwight David Eisenhower; la "Alianza para el Progreso" diseñada por John F. Kennedy. En el caso de la URSS, el tema no es tan simple. Siguiendo las palabras de Kokoshin (1997), la doctrina militar en ese país estaba más comprometida con la propia coyuntura política internacional que con una serie de postulados específicos. En la práctica, consistía en un compendio de sobreentendidos, lo bastante flexible como para admitir en todo momento una lectura adaptada a las necesidades políticas coyunturales. 
esfera de influencia hacia Oriente. Como respuesta, el gobierno ruso amenazó con neutralizar las medidas de Estados Unidos con el despliegue de un sistema Iskander en la región de Kaliningrado, a pocos kilómetros de Polonia; paralelamente a la posibilidad de utilizar los recursos de la Fuerza Naval rusa con la misma finalidad.

b. El posible ingreso de Ucrania y Georgia a la OTAN, forman parte de la clásica estrategia estadounidense de "institucionalización de su influencia”. En este caso, la proyección del ingreso de estas dos naciones a la organización militar, le otorgaría a los Estados Unidos un plus que trasciende considerablemente los beneficios netamente militares, ya que implicaría el retiro final de ambas naciones de la órbita de influencia moscovita.

c. En la misma dirección, Estados Unidos ha venido implementado desde hace ya mucho tiempo, su clásica estrategia de alianzas institucionales con países en desarrollo, en este caso, con naciones del continente Latinoamericano. De diverso contenido ${ }^{9}$, estos acuerdos le han permitido al gobierno norteamericano extender su área de influencia sobre algunos países de la región.

d. El 24 de abril del 2008, el gobierno de Estados Unidos anunció la reactivación de la cuarta flota en Latinoamérica y el Caribe, luego de más de cinco décadas de inactividad. Con el supuesto objetivo de fortalecer las labores humanitarias, combatir el narcotráfico y el terrorismo, actualmente la formación naval al mando de Joseph Kernan, surca las aguas del continente. Pero más allá de las declaraciones oficiales de Washington, esta medida parece indicar que Estados Unidos ha decidido reaccionar frente a la progresiva pérdida de hegemonía en el continente.

e. Las nuevas relaciones bilaterales entre Rusia y Venezuela, amenazan con modificar la balanza de poder en el continente americano. El

$9 \quad$ Entre los acuerdos económicos se pueden destacar los TLC firmados con Chile, Colombia, Costa Rica, entre otros; al nivel de seguridad, el Plan Colombia, la Iniciativa Mérida, etc. fuerte acercamiento de estas dos naciones se hizo evidente a raíz de una serie de acontecimientos durante el último año: (I) la compra progresiva de armamento ruso por parte del gobierno venezolano. "A Rusia, Venezuela le ha comprado más de $\$ 5400$ millones en armas desde el 2005" (La Nación, 7 de noviembre del 2008); (II) el pasado 10 de septiembre, dos bombarderos supersónicos de la aviación estratégica rusa, Tupolev- $160^{10}$, aterrizaron en una base militar del norte de Venezuela con el aparente fin de realizar vuelos de adiestramiento; (III) los Gobiernos de ambos países realizaron a finales del 2008 maniobras militares conjuntas en las aguas del Caribe, a pocos kilómetros de Estados Unidos. "Rusia enviará una flota con cuatro barcos de guerra con un millar de hombres a bordo, entre ellos el crucero a propulsión nuclear "Pedro el Grande" y el "Almirante Shabanenko", un barco de ataque contra submarinos" (Clarín, 9 de septiembre del 2008); (IV) el pasado 11 de septiembre, el gobierno de Hugo Chávez decidió expulsar al embajador de Estados Unidos en el país, Patrick Duddy. Como represalia, el gobierno estadounidense declaró pocos días después que el embajador de Venezuela en Washington, sería expulsado en represalia por lo sucedido con su homólogo estadounidense en Caracas; (v) durante el tercer viaje de Hugo Chávez a Moscú en el 2008, el Kremlin anunció un préstamo de 1000 millones de dólares para la compra de armamento ruso, paralelamente, los representantes máximos de ambos países manifestaron su intención de intensificar la cooperación económica, militar, tecnológica y energética.

f. El 10 de septiembre del 2008, Evo Morales comunicó su decisión de expulsar al embajador estadounidense en la Paz, Philip

10 Estos aviones pueden cargar hasta 40000 kilos de bombas $y$ arsenal nuclear. Alcanzan una velocidad de $2000 \mathrm{Km}$. por hora y tiene la suficiente autonomía para volar más de $15000 \mathrm{Km}$. sin la necesidad de recargar combustible, lo suficiente para volar desde Rusia a los Estados Unidos ida y vuelta. 
Goldberg, acusándolo de persona no grata y de animar el separatismo en el país. Estados Unidos respondió a los pocos días con la misma medida, declarando al diplomático boliviano en Washington, Gustavo Guzmán, persona no grata.

g. El nuevo acercamiento entre Nicaragua $y$ el Kremlin fue cristalizado por el anuncio de una visita oficial de delegados rusos en el país centroamericano. Entre los proyectos a implementar, el ministro ruso en Nicaragua destacó los siguientes: exploración de petróleo; explotación agrícola; construcción de infraestructura; reposición de armamento de guerra; cooperación educativa y la posibilidad de implementar un proyecto para la construcción de un canal interoceánico.

h. La reactivación de la cooperación militar entre Rusia y Cuba, quizá sea el ejemplo más representativo de la nueva orientación de la política exterior del Kremlin. A escasos kilómetros de las costas estadounidenses, Rusia estaría dispuesta a recuperar el control de uno de sus Estados satélites más férreos durante la Guerra Fría. Según fuentes rusas, ya hay programada una visita oficial a Cuba por parte del jefe de los cuarteles generales de la Defensa Aérea, teniente general Alexander Maslov, con el propósito de "intercambiar su experiencia en organización táctica de defensa aérea y en el entrenamiento de soldados" (El País, 29 de octubre del 2008).

i. Otra serie de acontecimientos, de menor trascendencia internacional, parecen indicar que la influencia estadounidense en la región estaría en decadencia: las duras declaraciones de la Cancillería argentina contra el gobierno de Estados Unidos a raíz del juicio por el contrabando de dinero; las sorpresivas críticas contra el proceder internacional de los Estados Unidos por parte del presidente costarricense Óscar Arias; las duras acusaciones del presidente boliviano, Evo Morales, contra la Oficina Antidrogas de Estados Unidos (DEA), a raíz del supuesto apoyo de Washington para el desarrollo del narcotráfico en el país andino; las acusaciones del presidente de Honduras contra Estados Unidos, entre otros.

Si bien ambos países parecen haber manifestado nuevamente su intención por extender su esfera de influencia en el mundo entero, ciertos patrones de comportamiento marcan una ruptura con el pasado. Históricamente, ambas potencias se preocuparon inicialmente por garantizar su supremacía y liderazgo en áreas geográficamente próximas a sus territorios, para posteriormente incursionar sobre aquellas regiones más aisladas. Sin embargo, a excepción de un par de oportunidades, ninguna superpotencia cedió deliberadamente el control de un territorio bajo su dominio sin mostrar resistencia previamente. En los últimos tiempos la situación parece haberse desplazado hacia una "lógica de influencia cruzada" (Fig. 1.2): Rusia ha perdido el control de muchas de las ex repúblicas socialistas soviéticas, pero se ha abocado fuertemente a la tarea de ampliar su influencia en el continente americano, históricamente feudo de la dominación estadounidense; por el otro lado, Estados Unidos ha cedido parcialmente su autoridad en su propio continente, pero ha buscado progresivamente ganarse el apoyo de muchas de las ex repúblicas socialistas soviéticas.

Si bien estos ejemplos no agotan la gran variedad de manifestaciones de Rusia y Estados Unidos por extender sus respectivas esferas de influencia, constituyen una muestra fehaciente de que ambas naciones han decidido revalidar su hegemonía en muchas regiones del planeta. En el caso de Estados Unidos, la desaparición de la URSS había frenado parcialmente esta iniciativa, al considerar que la victoria sobre el comunismo le había garantizado su supremacía sobre cualquier nación del planeta. Sin embargo, el conflicto desarrollado en Georgia, motivó la reaparición de Rusia. Actualmente, Moscú parece haber decidido recuperar mucho del terreno perdido durante la década de los noventa. 


\section{"LÓGICA DE INFLUENCIA CRUZADA"}

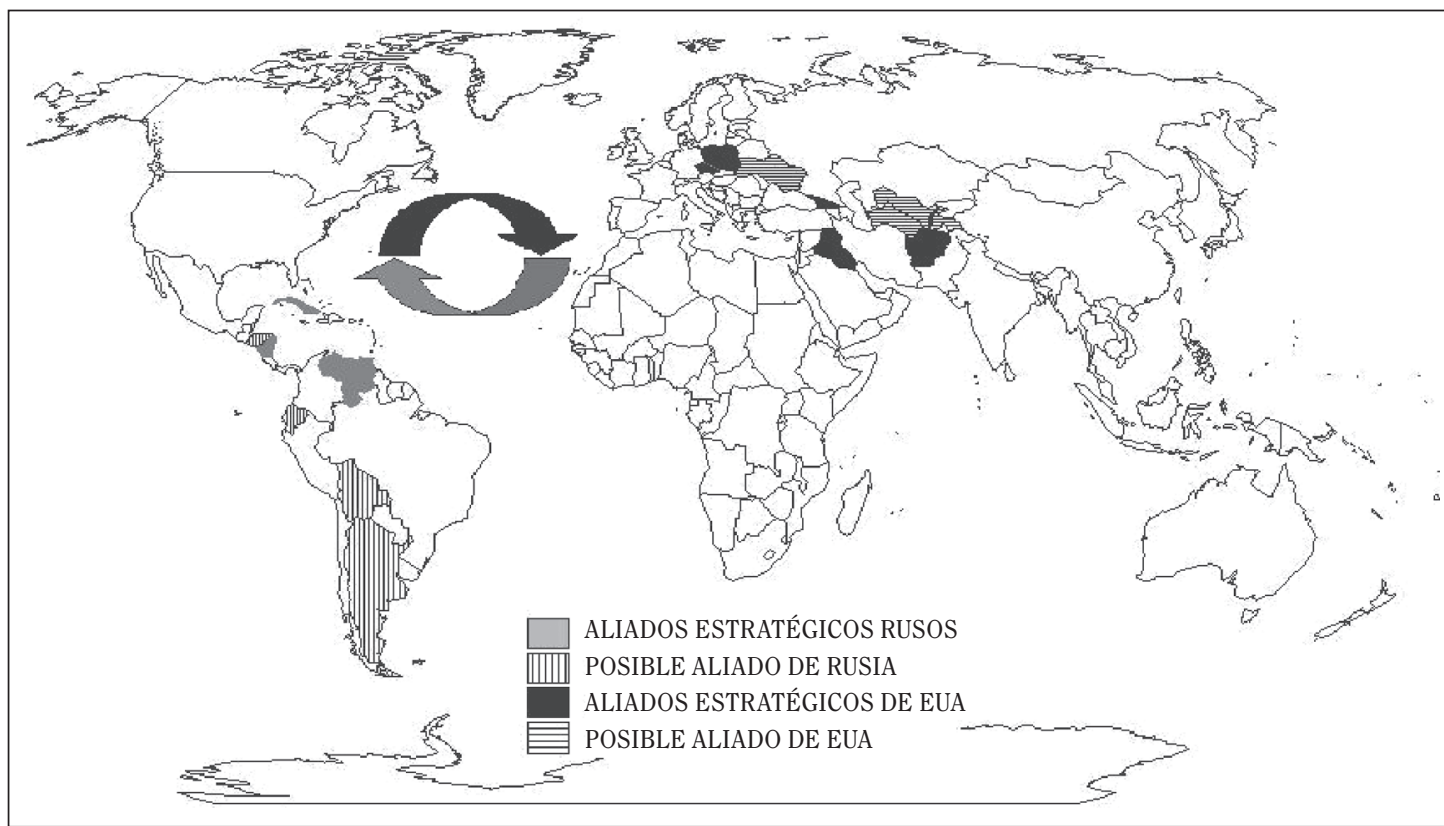

Fuente: Autor.

\section{CONCLUSIONES}

La Geopolítica se ha caracterizado, entre sus disciplinas afines, por su carácter eminentemente estático. Con un poco más de un siglo de existencia, muchos de sus postulados clásicos han prevalecido, casi sin variaciones, hasta el presente. Fundamentalmente un factor ha contribuido en esta dirección: la producción intelectual en la Geopolítica ha sufrido un fuerte revés debido a ser identificada continuamente como el "actor intelectual" de los grandes estragos durante las guerras mundiales, transformándose en un tabú dentro de los ámbitos académicos y políticos internacionales. Sin embargo, durante los últimos años, un grupo de intelectuales ha venido promoviendo fuertes trasformaciones dentro de esta disciplina. La Escuela Crítica ha cuestionado muchos de sus fundamentos, en una misión emancipadora del legado intelectual geopolítico clásico. Este conjunto de investigadores ha rechazado sistemáticamente "las verdades irrefutables" de la disciplina, en un intento por deconstruir y hacer una ruptura en sus bases teóricas más trascendentales.

Todo parecía indicar que la coyuntura internacional había evolucionado en una dirección completamente nueva. Sin embargo, una serie de hechos inesperados retrotraerían el statu-quo a unas cuantas décadas atrás. Las continuas intervenciones norteamericanas en el antiguo territorio soviético, motivaron la reaparición imperialista rusa. Como resultado, ambas potencias decidieron - con mayor firmeza durante el 2009- reactivar un conjunto de códigos geopolíticos característicos de otra época, que si bien no fueron los únicos, han distinguido la episteme durante la Guerra Fría. La ingeniería teórica elemental de estos códigos geopolíticos encuentra sus ondas raíces en el pensamiento geopolítico tradicional: la teoría del Corazón Continental (Heartland) de Halford Mackinder, la teoría de la Contención de George Kennan y el paradigma de las Esferas de Influencia.

Hemos observado como cada uno de ellos ha estado presente en la configuración 
de las políticas exteriores de Rusia y Estados Unidos durante los últimos años. En primer lugar, la influencia de la tesis mackinderiana del Heartland ha sido contrastada por la gran cantidad de intervenciones norteamericanas en la región del "cinturón interior". Tal cual vimos en los ejemplos, Rusia manifestó sorpresivamente una conducta indiferente en un primer momento, pero luego del estallido del conflicto en Georgia (2008), el Kremlin modificó su comportamiento radicalmente. En segundo lugar, Estados Unidos parece haber implementado una nueva versión de la clásica política de Contención frente al avance ruso, aunque esta tendencia parece haber disminuido su paso en los últimos meses. Por último, resulta evidente que ambas potencias han decidido reivindicar sus esferas de influencia en numerosos enclaves del planeta. En lo que aquí denominamos "lógica de influencia cruzada", Estados Unidos y Rusia se han abocado a la tarea de extender su dominio en el "patio trasero" de su antiguo adversario.

Las tres teorías ${ }^{11}$ han sido objeto de una nueva revalidación empírica. Es evidente que en los últimos años, Estados Unidos y Rusia decidieron recurrir nuevamente a ellas en la configuración de sus respectivos códigos geopolíticos. Siguiendo a Agnew, podríamos afirmar que la presencia de muchos de estos postulados de la Geopolítica tradicional, fue el resultado de las construcciones sociales de las elites políticas de los países más poderosos del planeta, en su afán por legitimizar las prácticas de sus respectivos Estados. Queremos evitar caer en conclusiones reduccionistas al afirmar que todos estos acontecimientos fundamentaron una nueva edición de la Guerra Fría. El sistema internacional es demasiado complejo como para encasillar los procesos más recientes de acuerdo con los modelos y esquemas del pasado. Sin embargo, no podemos evitar trazar fuertes similitudes con el ayer: los actores, los procedimientos y la estructura del sistema internacional presentaron características por lo menos comparables con la Guerra Fría.

11 Tal cual expusimos previamente, en el caso de las Esferas de Influencia no es posible identificar una base teórica específica que la sustente.

\section{BIBLIOGRAFÍA}

Agnew, John; Mitchell, Katharyne and ó Tuathail, Gerard. A companion to Political Geography. 1ra Edición. Estados Unidos: Blackwell Publishing, 2008.

Agnew, John (1998). Geopolítica. Una re-visión de la política mundial. 3ra Edición. España: Trama editorial, 2005a.

Agnew, John; Corbridge, Stuart. Mastering space. Hegemony, territory and international political economy. 1ra Edición. Estados Unidos: Routledge, 1995b.

Bush, George W. "Operation Iraqi Freedom". President Discusses Beginning of Operation Iraqi Freedom. Estados Unidos: The White House oficial web, March 22, 2003. En: <http://www.whitehouse.gov/ news/releases/2003/03/20030322.html> [consultado 22 de diciembre 2008].

Cairo, Heriberto; Pastor, Jaime (editores). Geopolítica, guerra y resistencias. 1era Edición. España: Trama Editorial, 2006.

Cox, Robert. Approaches to World Order. 1era Edición. Estados Unidos: Cambridge University Press, 1996.

Fucuyama, Francis (1992). The End of History and the Last Man. 3era Edición. Estados Unidos: Free Press, 2006.

Gaddis, J. L. Strategies of Contaiment. lera Edición. Estados Unidos: Oxford University Press, 1982.

Huntington, Samuel (1997). El choque de civilizaciones. 7ma Edición. Argentina: Paidós, 2004.

Kennan, George. "Las fuentes de la conducta soviética”. Foreign Affairs 25 (4). 1947: 566-582. 
Kokoshin, Andrei A. Soviet Strategic Thought, 1917-91. 1ra Edición. Estados Unidos: MIT Press, 1998.

Mackinder, Halford. "El pivote geográfico de la Historia". The Geograpfhical Journal XXIII (4). 1904: 21 y siguientes.

Ó Tuathail, Gearóid. Critical Geopolitics. 1ra Edición. Estados Unidos: University of Minnesota Press, 1996.

Palacios, José Miguel; Arana, Paloma. "Doctrina militar rusa: herencia soviética, realidades postsoviéticas, perspectiva europea". Revista CIDOB d'Afers Internacionals 59. 2005: 54-78.

Ratzel F. y otros. Antología Geopolitica. 1ra Edición. Argentina: Pleamar, 1975.

Taylor, Peter; Flint, Colin (1994). Geografía política. Economía mundo, Estadonación y localidad. 2da Edición. España: Trama Editorial, 2002.

DIARIOS

La Nación digital. "Gobierno de Bolivia acusa a la DEA de fomentar el narco". 7 de noviembre del 2008 . En: <http://www.nacion.com/ln_ee/2008/ noviembre/07/mundo1765957.html> [consultado 12 de noviembre 2008].

La Nación digital. "Venezuela anuncia que seguirá comprando armas". 7 de noviembre del 2008. En: <http://www. nacion.com/ln_ee/2008/noviembre/07/ mundo1765955.html>_[consultado $10 \mathrm{de}$ noviembre 2008].

La Nación digital. "Rusia amenaza con movilizar misiles cerca de Polonia". 6 de noviembre del 2008. En: <http://www. nacion.com/ln_ee/2008/noviembre/06/ mundo1764468.html> [consultado 7 de noviembre de 2008].
El País digital. "Cuba y Rusia reactivan la cooperación militar como parte de la intensificación de sus relaciones”. 29 de octubre del 2008. En: <http://www. elpais.com/articulo/internacional/ Cuba/Rusia/reactivan/cooperacion/ militar/parte/intensificacion/relaciones/ elpepuint/20081029elpepuint_3/Tes> [consultado 1 de noviembre 2008].

El País digital. "Polonia y República Checa aceptan el escudo antimisiles de EE UU". 28 de octubre del 2008. En: $<$ http://www.elpais.com/articulo/ internacional/Polonia/Republica/Checa/ aceptan/escudo/antimisiles/EE/UU/ elpepuint/20070220elpepiint_12/Tes> [consultado 1 de noviembre 2008].

La Nación digital. "Diario de EE. UU. critica a aliados de Chávez". 7 de octubre del 2008. En: <http://www.nacion.com/ ln_ee/2008/octubre/07/mundo1728165. html> [consultado 8 de octubre 2008].

La Nación digital. "Nos sorprendió apoyo tácito de Arias a Cuba y Chávez". 28 de septiembre del 2008. En: <http://www. nacion.com/ln_ee/2008/septiembre/28/ pais1606715.html> [consultado 29 de noviembre 2008].

Clarín digital. "Rusia le vendió armas a Venezuela y le ofreció ayudarla a desarrollar energía nuclear". 25 de septiembre del 2008. En: <http://www. clarin.com/diario/2008/09/25/um/m01767970.htm > [consultado $27 \mathrm{de}$ septiembre 2008].

El Nuevo Diario digital. "Rusia renovará armamento de ejército nicaragüense". 24 de septiembre del 2008. En: <http://www. elnuevodiario.com.ni/politica/27809> [consultado 26 de septiembre 2008].

Corriere della Sera digital. "Drone russo abbattuto in Georgia". 23 de septiembre 
del 2008. En: <http://www.corriere.it/ esteri/08_settembre_23/drone_russo_ abbattuto_fc7a2e7a-894a-11dd-823500144f02aabc.shtml> [consultado 24 de septiembre de 2008].

Clarín digital. "Barcos militares rusos viajan a Venezuela". 22 del septiembre del 2008. En: <http://www.clarin.com/ diario/2008/09/22/um/m-01765516.htm> [consultado 23 de septiembre 2008].

La Nación digital. "Rusos regresan a Nicaragua por crudo". 22 del septiembre del 2008. En: <http://www.nacion.com/ln_ee/2008/ septiembre/21/mundo1709173.html> [consultado 22 de septiembre 2008].

La Nación digital. "Cayó la coalición de gobierno pro occidental de Ucrania”. 17 de septiembre del 2008. En: <http://www. nacion.com/ln_ee/2008/septiembre/17/ mundo1703270.html> [consultado $17 \mathrm{de}$ septiembre 2008].

Corriere della Sera digital. "Russia, cresce la spesa per il riarmo". 16 de septiembre del 2008. En: $<$ http://www.corriere.it/esteri/08_ settembre_16/russia_difesa_armi_ putin_a 5ee 4 df6-83f6-11dd-8a6a00144f02aabc.shtml> [consultado 16 de septiembre 2008].

La Nación digital. "Arden las relaciones entre Estados Unidos y América Latina”. 12 de septiembre del 2008. En: <http://www. nacion.com/ln_ee/2008/septiembre/13/ mundo1698980.html> [consultado 12 de septiembre 2008].

La Nación digital. "EE.UU. expulsa al embajador de Venezuela como represalia". 12 de septiembre del 2008. En: <http://www. nacion.com/ln_ee/2008/septiembre/13/ mundo1698961.html> [consultado $13 \mathrm{de}$ septiembre 2008].
La Nación digital. "Bombarderos estacionados en Venezuela". 12 de septiembre de 2008. En: <http://www.nacion.com/ln_ee/2008/ septiembre/10/mundo1696826.html> [consultado 12 de septiembre 2008].

Corriere della Sera digital. "Chavez espelle l'ambasciatore Usa «Andate all'inferno, yankee di m...»". 12 de septiembre del 2008. En: <htp:// www.corriere.it/esteri/08_settembre_12/ chavez_usa_bolivia_6833d86e-80ae$11 \mathrm{dd}-\mathrm{b} 38 \mathrm{~b}-00144 \mathrm{f} 02 \mathrm{a} a \mathrm{bc} . \mathrm{shtml}>$ [consultado 13 de septiembre 2008].

Corriere della Sera digital. "Sarah Palin: «Guerra alla Russia? Forse»". 12 de septiembre del 2008. En: <http:// sitesearch.corriere.it/forward.jsp > [consultado 13 de septiembre 2008].

New York Times digital. "U.S. Calls Venezuelan Officials Rebel Supporters". 12 de septiembre del 2008. En: <http://www.nytimes.com/2008/09/13/ world/a meric a s / 13 venez. html? scp $=1 \& \mathrm{sq}=\mathrm{U} . \mathrm{S} . \% 20 \mathrm{Calls} \% 20$ Venezuelan\%200fficials\%20Rebel\%20 Supporters\%20\&st=cse> [consultado 13 de septiembre 2008].

La Nación digital. "Chávez afirma que aviones rusos en su país es un "aviso" al imperio". 11 de septiembre del 2008. En: <http://www.nacion.com/ln_ee/2008/ septiembre/11/mundo1698023.html> [consultado 11 de septiembre del 2008].

La Nación digital. "Bombarderos estratégicos rusos aterrizan en Venezuela". 11 de septiembre del 2008. En: <http://www. nacion.com/ln_ee/2008/septiembre/11/ mundo1696586.html> [consultado 12 de septiembre 2008].

Corriere della Sera digital. "Sud Ossezia: «Vogliamo far parte della Russia, non essere indipendenti»". 11 de septiembre 
del 2008. En: <http://www.corriere. it/esteri/08_settembre_11/ossezia_ entrare_russia_ce6abbd2-7fd9-11dd-9f6f00144f02aabc.shtml> [consultado 12 de septiembre 2008].

Corriere della Sera digital. "Chavez: «I bombardieri russi sono un avvertimento agli Usa»". 11 de septiembre de 2008. En: <http://www.corriere.it/esteri/08_ settembre_11/chavez_bombardieri_ russi_ade95136-8036-11dd-9f6f00144f02aabc.shtml> [consultado 12 de septiembre 2008].

Clarín digital. "Rusia hará ejercicios de guerra con su marina en el Caribe venezolano". 9 de septiembre de 2008. En: <http:// www.clarin.com/diario/2008/09/09/ um/m-01756382.htm> [consultado $10 \mathrm{de}$ septiembre 2008].

New York Times digital. "Russia and Venezuela Confirm Joint Military Exercises". 8 de septiembre del 2008. En: <http:// www.nytimes.com/2008/09/09/ world/americas/09venez.html? $\mathrm{r}=1 \& \mathrm{~s} \mathrm{c} \mathrm{p}=1 \& \mathrm{~s} \mathrm{q}=\mathrm{R}$ u s s i a $\% 20$ a n d \% $20 \mathrm{~V}$ e n e $\mathrm{z}$ u e 1 a $\% 20$ Confirm\%20Joint\%20 Military\%20 Exercises \& $\mathrm{st}=\mathrm{cse} \&$ oref $=\mathrm{s} \log$ in $>$ [consultado 9 de septiembre 2008].

New York Times digital. "U.S. Backs Off Civilian Nuclear Pact With Russia". 8 de septiembre del 2008. En: <http://www.nytimes. com/2008/09/09/washington/09nuclear. html ? scp $=1 \&$ sq $=$ U.S. $\% 20$ Backs $\% 20$ Off\%20Civilian\%20Nuclear\%20Pact $\% 20$ With\%20Russia\%20\&st=cse $>$ [consultado 9 de septiembre 2008].

Corriere della Sera digital. "Sarkozy: In Georgia 200 uomini» Truppe russe ritirate entro un mese". 8 de septiembre del 2008 . En: <http://www.corriere.it/esteri/08_ settembre_08/russia_missione_ue_ georgia_1698ba56-7d8e-11dd-8ad100144f02aabc.shtml> [consultado 9 de septiembre 2008].

La Nación digital. "EE. UU. alza el tono contra Rusia”. 5 de septiembre del 2008. En: <http://www.nacion.com/ln_ee/2008/ septiembre/05/mundo1689300.html> [consultado 7 de septiembre 2008].

Clarín digital. "EE.UU. dio nuevas muestras de apoyo a Ucrania". 5 de septiembre del 2008. En: <http://m.clarin.com/Luxe/ Noticia.aspx?IdNoticia $=01753796>$ [consultado 5 de septiembre 2008].

La Jornada digital. "Washington busca recobrar hegemonía en América Latina con la Cuarta Flota". 27 de agosto del 2008. En: <http://www.jornada.unam. $\mathrm{mx} / 2008 / 08 / 27 /$ index.php?section=politi ca\&article $=023 \mathrm{n} 1 \mathrm{pol}>$ [consultado $27 \mathrm{de}$ agosto 2008].

La voz de Asturias digital. "EEUU recibe luz verde para instalar su escudo antimisiles en Polonia”. 20 de agosto del 2008. En: $<w w w . l a v o z d e a s t u r i a s . e s>$ [consultado 21 de agosto 2008].

DW-World digital. "República Checa-EE.UU.: radar sin cohetes". 7 de agosto del 2008. En: <http://www.dw-world.de/ $\mathrm{dw} /$ article $/ 0,2144,3470260,00 . \mathrm{html}>$ [consultado 7 de agosto 2008].

BBC mundo digital. "Después de 58 años, la armada estadounidense reactivará la cuarta flota, encargada de patrullar los mares latinoamericanos". 8 de mayo del 2008. En: <http://news.bbc. co.uk/hi/spanish/latin_america/ newsid_7389000/7389057.stm> [consultado 9 de mayo 2008]. 
